\title{
ORIGINAL ARTICLE \\ Functional implications of corticospinal tract impairment on gait after spinal cord injury
}

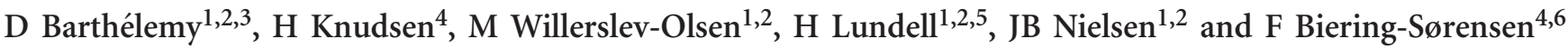

\begin{abstract}
Objective: Maximum toe elevation during walking is an objective measure of foot drop and reflects the impairment of the corticospinal tract (CST) in persons with spinal cord injury (SCI). To determine if this measurement is functionally relevant to ambulatory abilities, we correlated maximum toe elevation with clinical physiotherapy tests.
\end{abstract}

Setting: Cross-sectional study, laboratory and clinical settings.

Methods: A total of 24 individuals with SCI (American Spinal Injury Association (ASIA) Impairment Scale D) were recruited. Maximum toe elevation during the swing phase of treadmill gait was measured with a kinematic system. CST function was assessed in a sitting position by measuring the motor-evoked potentials (MEPs) induced in tibialis anterior muscle with transcranial magnetic stimulation over the motor cortex. Clinical tests performed were 10-m and 6-min walk test (6MWT), Timed-Up and Go (TUG), Walking Index for Spinal Cord Injury, Berg Balance Scale, Lower Extremity Motor Score (LEMS) and sensory score of the L4, L5 and S1 dermatomes.

Results: Participants with lower toe elevation during gait walked at a slower speed, took more time to perform the TUG test, and covered a shorter distance in the 6MWT. They also scored lower on the LEMS and showed impaired superficial sensitivity of the dermatomes around the ankles. Few correlations were observed between CST function and clinical tests, but the presence of MEP at rest was indicative of faster speed and longer distance in the 6MWT.

Conclusion: These results indicate that maximum toe elevation, which is directly correlated with CST impairment, is functionally relevant as it also correlates with timed clinical tests, LEMS and sensory scores.

Spinal Cord (2013) 51, 852-856; doi:10.1038/sc.2013.84; published online 13 August 2013

Keywords: spinal cord injury; corticospinal tract; transcranial magnetic stimulation; walking; clinical tests

\section{INTRODUCTION}

Technological developments in recent years have produced new tools for assessing motor deficits as the result of injury. Notably, transcranial magnetic stimulation (TMS) is used to assess the corticospinal tract (CST): the amplitude and latency of the motor-evoked potential (MEP) induced by TMS reflects impaired transmission in CST. Although CST transmission is increased following specific interventions, ${ }^{1,2}$ correlation between MEP characteristics and overall gait function is not all that clear. While motor function can remarkably improve during recovery, MEP latency remains pathologically prolonged. ${ }^{3}$ Also, a recent study demonstrated lack of correlation between measures of MEPs and gait. ${ }^{4}$ One reason might be that CST involvement is more prominent in discrete subtasks of the function rather than in the overall task, which requires concurrent input from many different systems. Identifying the precise role of CST within gait will enable a more specific assessment of incapacities following CST lesion. Previous studies and clinical assessment of stroke patients indicate that the CST is involved in foot elevation during swing and that its impairment induces foot drop. ${ }^{5-7}$ We have recently shown that measurement of maximum toe elevation during swing can be used as a simple and objective measurement of foot drop and reflects CST impairment in persons with spinal cord injury (SCI). ${ }^{8}$ Notably, MEP latency was correlated to maximum toe elevation but not to overground speed. In parallel, maximum toe elevation was correlated to overground speed, suggesting that a direct link between MEP characteristics and gait function might be difficult to establish. Maximum toe elevation may however constitute an indirect link between CST and overall gait function. Hence, in this paper, we hypothesize that clinical measures of gait function are correlated to maximum toe elevation, which in turn is directly correlated to CST impairment.

\section{PATIENTS AND METHODS}

Twenty-four participants with SCI took part in this study. Data were collected in a research laboratory at the University of Copenhagen and the physiotherapy department of the Clinic for Spinal Cord Injuries (Glostrup University Hospital, Hornbaek), Denmark. The experimental protocol was approved by the local ethics committee and was in accordance with the Declaration of Helsinki. Participants received information and gave their written consent to the study.

\section{Participants}

Two women and twenty-two men with SCI were recruited (age 20-65 years; mean of 43.4 years; Table 1 for a description of the participants). They al

${ }^{1}$ Department of Exercise and Sport Sciences, University of Copenhagen, Copenhagen, Denmark; ${ }^{2}$ Department of Neuroscience and Pharmacology, University of Copenhagen, Copenhagen, Denmark; ${ }^{3}$ School of Rehabilitation, Université de Montréal, Montreal, Quebec, Canada; ${ }^{4}$ Clinic for Spinal Cord Injuries, Glostrup University Hospital, Hornbæk, Denmark; ${ }^{5}$ Danish Research Centre for Magnetic Resonance, Copenhagen University Hospital, Hvidovre, Denmark and ${ }^{6}$ Faculty of Health Sciences, University of Copenhagen, Copenhagen, Denmark

Correspondence: Professor D Barthélemy, School of Rehabilitation, Faculty of Medicine, Université de Montréal, Pavillon du Parc, C.P.6128 Succ. Centre-ville, Montreal, Quebec, Canada H3C $3 \mathrm{~J} 7$.

E-mail: dorothy.barthelemy@umontreal.ca

Received 13 March 2013; revised 19 June 2013; accepted 27 June 2013; published online 13 August 2013 
Table 1 Description of spinal cord injured participants included in this study

\begin{tabular}{|c|c|c|c|c|c|c|c|c|}
\hline $\begin{array}{l}\text { Participant } \\
\text { number }\end{array}$ & $\begin{array}{l}\text { Male }(M) / \\
\text { female }(F)\end{array}$ & $\begin{array}{c}\text { Age } \\
\text { (years) }\end{array}$ & $\begin{array}{l}\text { Years } \\
\text { since } \\
\text { lesion }\end{array}$ & $\begin{array}{c}\text { Neuro- } \\
\text { logical } \\
\text { level }\end{array}$ & $A / S$ & $\begin{array}{l}\text { LEMS } \\
R(/ 25)\end{array}$ & $\begin{array}{c}\text { LEMS L } \\
\text { (/25) }\end{array}$ & $\begin{array}{c}B M I \\
\left(\mathrm{~kg} \mathrm{~m}^{-2}\right)\end{array}$ \\
\hline 1 & $M$ & 47 & 28 & C4 & D & 20 & 16 & 24.0 \\
\hline 2 & $M$ & 64 & 4 & $\mathrm{C} 3$ & D & 25 & 25 & 22.0 \\
\hline 3 & $M$ & 49 & 5 & L1 & $D$ & 25 & 23 & 24.4 \\
\hline 4 & $M$ & 28 & 1 & T12 & D & 21 & 21 & 19.7 \\
\hline 5 & $M$ & 32 & 3 & T5 & $D$ & 23 & 23 & 17.9 \\
\hline 6 & $\mathrm{~F}$ & 65 & 2 & C6 & D & 21 & 22 & 26.2 \\
\hline 7 & $M$ & 21 & 2 & $\mathrm{C} 2$ & D & 22 & 25 & 21.7 \\
\hline 8 & $M$ & 44 & 9 & T12 & $A(D)^{a}$ & 15 & 24 & 34.7 \\
\hline 9. & $M$ & 50 & 6 & $\mathrm{C} 2$ & D & 13 & 25 & 27.3 \\
\hline 10 & $M$ & 62 & 30 & $\mathrm{C} 4$ & $D$ & 20 & 13 & 22.0 \\
\hline 11 & $M$ & 38 & 21 & $\mathrm{C} 2$ & $\mathrm{D}$ & 12 & 23 & 23.8 \\
\hline 12 & $M$ & 48 & 14 & $\mathrm{C} 1$ & $D$ & 24 & 25 & 22.7 \\
\hline 13 & $M$ & 45 & 22 & C5 & $D$ & 23 & 25 & 24.1 \\
\hline 14 & $M$ & 31 & 9 & C5 & D & 49 & 50 & 22.7 \\
\hline 15 & $M$ & 62 & 38 & $\mathrm{C} 4$ & $D$ & 22 & 24 & 24.7 \\
\hline 16 & $M$ & 32 & 1 & C5 & D & 25 & 25 & 25.8 \\
\hline 17 & $M$ & 54 & 13 & C5 & D & 23 & 25 & 27.7 \\
\hline 18 & $M$ & 45 & 24 & C4 & $\mathrm{D}$ & 43 & 44 & 25.7 \\
\hline 19 & $M$ & 42 & 25 & $\mathrm{C} 3$ & $D$ & 43 & 34 & 29.0 \\
\hline 20 & $M$ & 20 & 1 & C5 & $D$ & 43 & 50 & 24.6 \\
\hline 21 & $M$ & 49 & 14 & T11 & $\mathrm{D}$ & 50 & 45 & 25.7 \\
\hline 22 & $M$ & 24 & 1 & $\mathrm{C} 2$ & $D$ & 50 & 48 & 23.5 \\
\hline 23 & $M$ & 28 & 8 & $\mathrm{C} 2$ & $D$ & 50 & 50 & 29.5 \\
\hline 24 & $\mathrm{~F}$ & 61 & 9 & $\mathrm{C} 2$ & D & 48 & 45 & 22.6 \\
\hline
\end{tabular}

Abbreviations: AIS, American Spinal Injury Association Impairment Scale; BMI, body mass index; LEMS, lower extremity motor score.

asee text for explanation.

sustained an incomplete SCI, which induced different levels of locomotor impairment (American Spinal Injury Association (ASIA) Impairment Scale (AIS) D). One participant lacked sacral sparing ${ }^{9}$ but could still walk and was otherwise equivalent in motor function to an AIS D participant. He was therefore included. Other inclusion criteria were that the spinal cord lesion (traumatic or nontraumatic) had to be stable for at least 1 year before the experiment and that there were no known peripheral neuropathies or other injuries that might interfere with gait. As for medication, four SCI participants took pain medication, two took medication for bladder control and five were taking antispastic medication. We also recruited 11 healthy individuals paired for age and gender to the SCI participants (10 men and 1 woman; age 22-67 years; mean 45 years) as control for normal values of highest toe elevation.

\section{Experimental procedures}

Kinematic recording. All laboratory procedures used in this paper were described in details in Barthélemy et al. ${ }^{8}$ Briefly, gait ability of participants was assessed on a treadmill (TechnoGym, Køge, Denmark) for 5 min at their maximum comfortable speed. SCI participants did not wear any orthosis during the assessments. They did wear a security harness to prevent falls, but it was not used for body-weight support.

The kinematics of locomotor movements was analyzed using a six-camera ProReflex Qualisys system (Qualisys, Gothenburg, Sweden). Passive markers were placed on both legs and were positioned on the anterior superior iliac spine, greater trochanter, external joint line of the knee, external malleolus and the lateral side of the fifth metatarsophalangeal joint (MTP). A pressuresensitive resistance sensor was placed under the heel of the most impaired leg to determine heel contact.

The kinematic recordings from the infrared cameras were preanalyzed with Qualisys software and then periods of 30-60s of measurements were exported to custom-made software (MATLAB and Excel-based) to determine maximum amplitude of toe elevation during swing. Toe amplitude was calculated from the distance between the marker placed on the MTP and the ground. The largest distance between these two points was determined and will be referred to as the maximum toe elevation. In healthy participants, plotting the elevation against time reveals the presence of two separate peaks of toe elevation during swing (see Barthelemy et al. ${ }^{8}$ ). Typically, a toe elevation was detected at swing onset and just before heel strike (first and second toe lifts, respectively; Figure 1).

The presence or absence of the second toe elevation before heel strike was also determined by subtracting the excursion of the malleolus marker (which can be caused by flexion of the knee, hip or other compensation strategies used by the SCI participants) from toe elevation. The remaining movement would then mainly occur at the ankle at a time corresponding to the second toe elevation.

Electrophysiological assessment. Only 20 participants (18 men and 2 women) were assessed in this part, as the other four participants presented exclusion criteria for TMS (seizures, fracture or metal implanted in the cranium). Electromyography activity was recorded from the Tibialis Anterior (TA) muscles of both legs. The signals were amplified (1000-5000), band-pass filtered $(5-25$ to $1000 \mathrm{~Hz})$, then digitized and sampled $(2 \mathrm{kHz})$ on a computer using a Microl401 interface (Signal software; Cambridge Electronic Design, Cambridge, UK).

The MEP amplitude was measured with the participant at rest in a sitting position with a hip angle of $90^{\circ}$ to $100^{\circ}$ and knee and ankle angles of $90^{\circ}$. The peak-to-peak value of each MEP was calculated, averaged and expressed as a percentage of the maximum compound muscle action potential (Mmax) elicited by a supramaximal electrical stimulation of the common peroneal nerve.

The latency was measured from the rectified and averaged MEP, during a contraction equivalent to $10 \%$ of the maximum voluntary contraction of the TA. The latency corresponded to the onset of the first lasting deviation above background electromyography.

Clinical assessment. A physiatrist, an experienced physical therapist and a researcher collected all data. Each evaluator was deliberately blinded to the outcome of the evaluation of the other evaluators before their own assessment (except for the AIS grade). The physiatrist performed the International Standards for the Neurological Classification of Spinal Injury (ISNCSCI) assessment, including AIS grade, and from these assessments determined the level of the spinal cord lesion. ${ }^{9}$ The lower extremity motor score (LEMS) is the manual assessment of motor strength of five key muscle functions in the limb (each muscle function is tested on a $0-5$ scale for a maximum of 25 for one lower limb). Sensory testing was also performed during this assessment for light touch and pinprick, with scores of 0 (no sensibility), 1 (impaired) and 2 (normal) for each dermatome.

The trained physical therapist assessed the participants in the following clinical tests according to the guidelines of the Spinal Cord Injury Rehabilitation Evidence (SCIRE; http://www.scireproject.com/). (1) Overground speed was determined on the basis of the 10 -m walking test: the distance $(10 \mathrm{~m})$ was divided by the time it took (in seconds) for the participant to walk that distance at their preferred speed. (2) The 6-min walking test (6MWT) measures the distance that a patient can walk in $6 \mathrm{~min}$ and has been validated for the SCI population. ${ }^{10}$ (3) Timed-Up and Go (TUG) is a validated test in which the participant, from sitting in a chair, stands up, walks $3 \mathrm{~m}$ at their preferred walking speed, returns to the chair and sits down. ${ }^{11}$ Participants were instructed to perform two TUGs, one with a left turn and another one with a right turn. We then took the mean of the time of the two TUGs to determine the mean TUG. (4) The Walking Index for Spinal Cord Injury II (WISCI II) is a validated scale used to assess the amount of physical assistance, braces or devices required to walk $10 \mathrm{~m}$. This scale is out of 20 , where 0 represents a patient unable to stand and 20 a person that ambulates with no device, brace or assistance. ${ }^{12}$ (5) The Berg Balance Scale (BBS) is a measure of balance that includes 14 tasks that are progressively more challenging for balance. Each task is scored from 0 (unable to perform) to 4 (normal) for a total possible score of 56. It was also validated for the SCI population. ${ }^{13,14}$ 

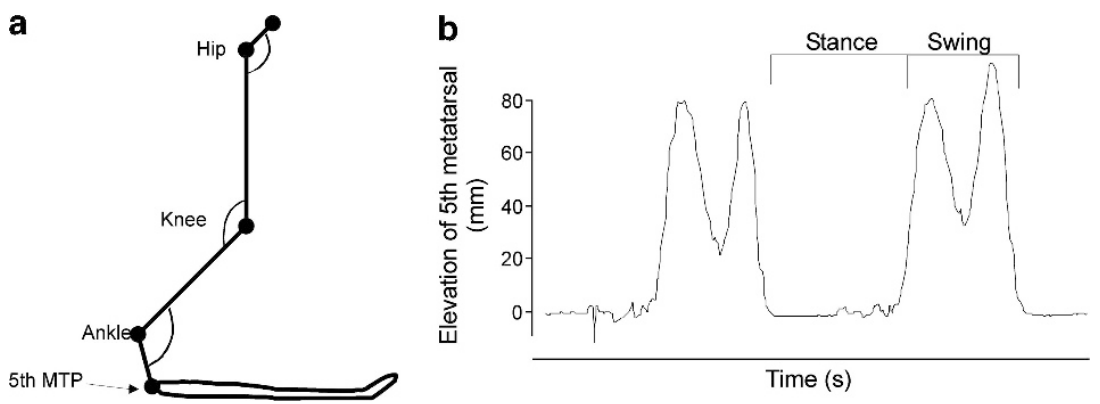

Figure 1 Measurement of toe elevation. (a) Reconstruction of the leg of a control participant based on markers put on bony landmarks. Angle of the hip, knee and ankle joint joints can be calculated and two toe elevations can be identified during swing. (b) The two toe elevations from a can be plotted on a graph where $x$ is the time and $y$ the elevation in $\mathrm{mm}$.

\section{Statistical analysis}

SigmaPlot11 was used for statistical analyses. Descriptive statistics on patient characteristics and outcome measurements were performed, followed by linearity assessment of the data with scatter plots. Normal data distribution was confirmed with the Kolmogorov-Smirnov test. Pearson's product-moment correlation was then used to assess correlations between the timed and measured outcome measurements. Data from ordinal scales (BBS, WISCI and LEMS as well as the motor and sensory scores of the ISNCSCI) were assessed with Spearman's $\rho$ coefficient. Student's $t$-test was used to perform group comparisons. Statistical significance was reached when $P<0.05$.

\section{RESULTS}

\section{Toe-elevation measurements}

Figure 1 describes the pattern of foot elevation during gait in a healthy individual. Two toe elevations can be observed: one at the onset of swing to lift the foot off the floor and the second one at the end of swing to prepare heel contact with the floor (Figure 1a and b). SCI participants showed a variety of patterns that we subgrouped into three distinct patterns. Thirteen SCI participants showed a biphasic toe elevation similar to control. The amplitude of these two elevations, however, could greatly vary. Five more impaired participants showed only the first toe elevation; the second elevation was not well defined and could not be identified as such, or was absent. A third subgroup $(n=6)$ exhibited a clear foot drag at the onset of swing. ${ }^{8}$ For all subjects, the maximum toe elevation during swing was calculated and used in the following correlations.

\section{Clinical tests}

Maximum toe elevation was strongly correlated to timed clinical tests such as speed (Figure 2a; $r=0.691, P<0.001$ ), 6MWT (Figure 2b; $r=0.573, P=0.004$ ) and TUG (Figure $2 c ; r=0.48, P=0.02$ ). For WISCI and BBS, a clear correlation could not be drawn due to a data ceiling effect, where 20 and 56 were the highest scores for those tests, respectively (Figures $2 \mathrm{~d}$ and $\mathrm{f}$ ). We then assessed if maximum toe elevation could be correlated to the LEMS (Figure 2e). Correlation could be observed with whole-leg motor score $(r=0.529 ; P=0.01)$, and particularly with the ankle dorsiflexor muscles $(r=0.613$; $P=0.002$ ). Furthermore, maximum toe elevation was also correlated to the sensory scores of the L4, L5 and S1 dermatomes $(r=0.618$, $P=0.003)$, which reflects superficial sensitivity around the ankle.

\section{Electrophysiological measures}

Maximum toe elevation in all participants was correlated to MEP latency and amplitude at $\operatorname{rest}^{8}$ (Table 2). MEP latency, however, was not correlated to gait function measured by clinical tests, or to the motor and sensory scores from the ISNCSCI. MEP amplitude at rest was correlated to speed $(r=0.7 ; P=0.009)$ and the 6MWT $(r=0.584 ; P=0.02)$. Correlation with the 6MWT did not follow a linear distribution; rather participants with an absence of MEP at rest covered less distance in the 6MWT than those who presented an MEP at rest ( $242 \pm 106 \mathrm{~m}$ vs $467 \pm 110 \mathrm{~m}$, respectively; Student's $t$-test $P=0.002)$.

\section{DISCUSSION}

These data show that the measurement of maximum toe elevation, which reflects CST impairment, is also functionally relevant, as it is correlated to speed and timed clinical tests. Correlation with WISCI and BBS were inconclusive probably due to a data ceiling effect, reported previously. ${ }^{14-16}$ Correlation with muscle strength assessed by LEMS was significant, but its interpretation limited due to the steplike shape of this scale. Strength assessment by measuring muscle force would confirm the correlation reported here. Moreover, maximum toe elevation was also correlated to ankle sensitivity, which suggests that foot drop is also dependent on intact sensory information. Hence, there is a direct link between maximum toe elevation and gait function.

\section{MEP measures correlate well to maximum toe elevation but not to} functional tests: an indirect link?

MEP latency was well correlated to maximum toe elevation but not to any of the timed clinical tests. One explanation is that we only assessed transmission in CST to TA of the most impaired leg, and gait involves more neuronal pathways than CST transmission to TA. TA MEPs are probably a reasonable measure of impaired CST transmission to ankle muscles, but does not provide information as to whether CST transmission to hip and knee muscles is also impaired. Furthermore, on the basis of animal experiments, other descending pathways than the CST are probably more important for gait function. As these pathways are not located in the same part of the spinal cord as the CST, the MEP measures do not provide relevant information about their impairment. Finally, the present data also showed that sensory information is important for gait function and evaluation of CST transmission would provide little information of this. MEP amplitude at rest was correlated to maximum toe elevation, to speed and to $6 \mathrm{MWT}$, but not in a linear fashion. Rather, the presence of an MEP at rest was associated with faster speed and covering more distance in the 6MWT. The presence of MEP amplitude at rest thus seemed indicative of gait-function recovery. Therefore, CST would be involved in gait function through control of toe elevation, foot trajectory and foot drag; our results indicate that, with proper foot control, speed increases and gait is more functional. Thus, by controlling foot drop, CST might be directly involved in enabling proper gait function. 

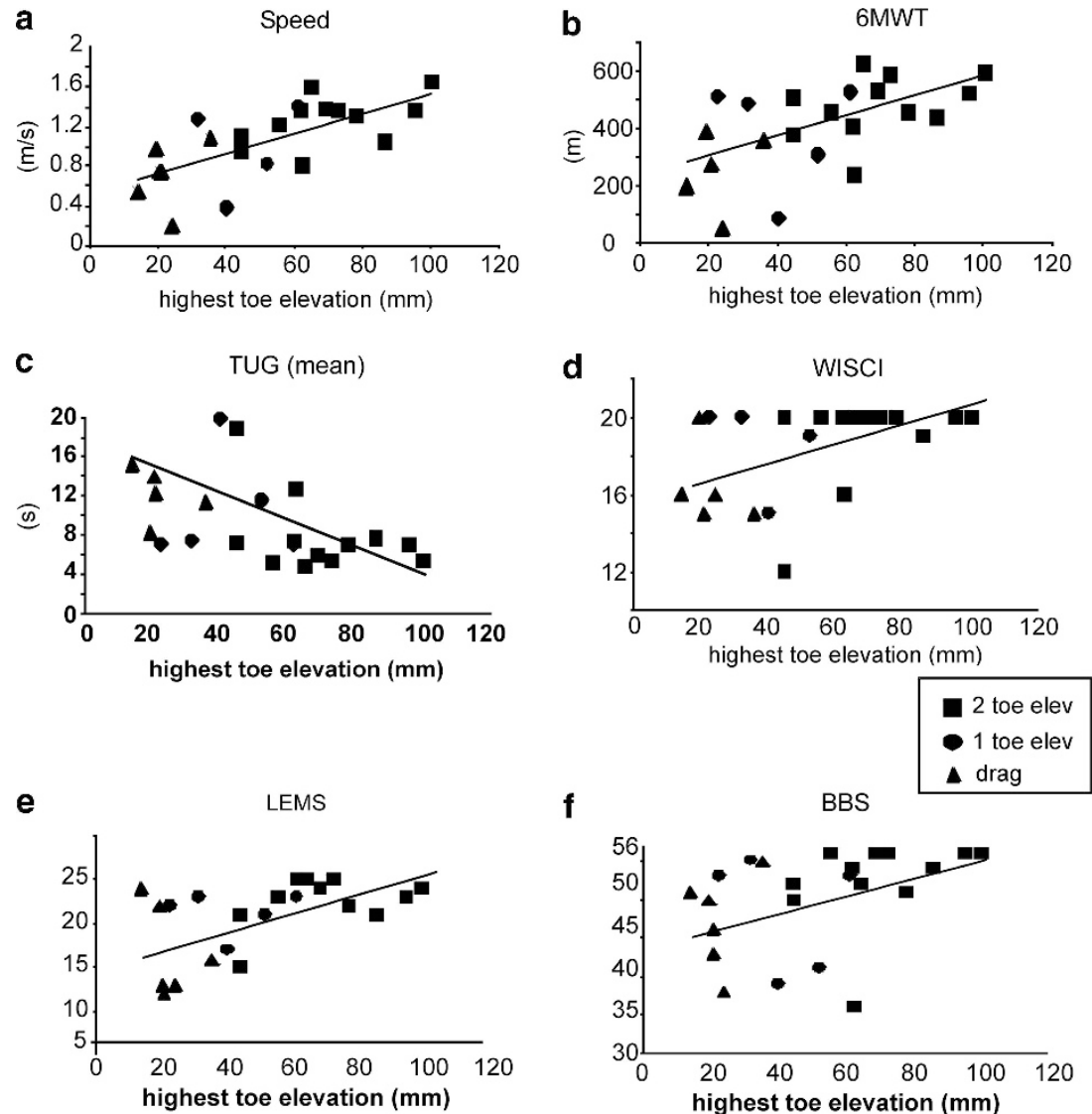

Figure 2 Correlations between maximum toe elevation and clinical tests. Graphs of correlations between maximum (highest) toe elevation and (a) speed (10-m test), (b) 6MWT, (c) TUG, (d) WISCI II, (e) LEMS and (f) BBS.

Table 2 Correlations between electrophysiological measures and clinical assessment of gait function

\begin{tabular}{lcccc}
\hline & $\begin{array}{c}\text { Maximal } \\
\text { toe elevation }\end{array}$ & $\begin{array}{c}\text { Speed: } \\
\text { 10- } m \text { test }\end{array}$ & $\begin{array}{c}\text { Timed-Up } \\
\text { and Go }\end{array}$ & $\begin{array}{c}6 \text {-min } \\
\text { walk test }\end{array}$ \\
\hline MEP latency & $r=0.5$ & $r=0.3$ & $r=0.3$ & $r=-0.3$ \\
MEP amplitude rest & $P=0.04^{*}$ & $P>0.05$ & $P>0.05$ & $P>0.05$ \\
& $r=0.6$ & $r=0.7$ & $r=-0.4$ & $r=0.58$ \\
& $P=0.01^{*}$ & $P<0.01^{*}$ & $P>0.05$ & $P=0.02^{*}$ \\
\hline
\end{tabular}

Abbreviation: MEP, motor-evoked potential. ${ }^{*} P<0.05$

\section{Foot-drop measurements}

Maximum toe elevation has been used previously ${ }^{17-19}$ and is a simple measurement for culling out the effect of CST lesion on gait function. It clearly reflected CST impairment, although compensations used by the participants to overcome foot drop were probably not completely excluded from those measurements. While many efforts were deployed to decrease all possible compensation that the participants had developed, those solutions were labor intensive and not all that informative or useful. Notably, we first tried to calculate compensation at other joints (e.g., hyperflexion of the ipsilateral hip or knee) and subtracting them systematically in every participant. ${ }^{8}$ That, however, was variable and inconclusive for the first toe elevation, even though subtraction of the malleolus marker from that of toe enabled assessment of the second toe elevation. We therefore opted to record the maximum toe elevation and prevented the participants from supporting themselves with their hands as much as possible, as this would facilitate certain types of compensation: by using the hand support as a fixed point, some participants were able to create a momentum at the knee and would 'swing' their leg forward, which would passively lift the toe. It was thus important to prevent that support, although it was not possible in a few cases. Furthermore, as the TA is an inverter of the foot as well as a dorsiflexor, the toe elevation induced might have been higher if it was measured on the medial side of the foot (elevation of the first MTP instead of the fifth MTP), thus giving the toes more clearance than what was observed in this study.

Although maximum toe elevation is relatively simple, some rehabilitation settings might not have access to the cameras necessary for kinematic analysis. Other tools could be used to quantify foot drop, such as electronic goniometers to assess ankle angle, which has also been used. ${ }^{8}$ Because of the compensation strategies exhibited by the participants in the study, ankle angle was not found to be as valid and reliable as maximum toe elevation. Notably, total ankle motion range could be troublesome as some participants had a decreased range (minimal movement at the ankle), whereas others had greater range than controls (notably through foot drag). We previously used ankle angle to assess excursion of the first part of swing (first toe elevation), ${ }^{8}$ but while this measure was correlated to speed, it was not correlated to any of the electrophysiological measurements. Nonetheless, other studies have assessed maximum dorsiflexion ${ }^{20}$ and ankle dorsiflexion at heel (or foot) contact. ${ }^{21}$ These possibilities should be further explored. 


\section{Why foot-drop measurements should be used in clinics}

There are many reasons why gait function, as assessed by clinical tests, could be impaired after SCI. Notably, it could be due to poor balance control, decreased strength at either proximal or distal part of the body and so on. Here, we identified that SCI with impaired CST would have decreased toe elevation, which would, in turn, have an impact on their speed as well as on their performance in validated clinical tests. Thus, this measurement could refine the assessment made of the patient and could guide therapists toward a better, more precise identification of the problem: CST impairment. Furthermore, although simple observation of the patient's dorsiflexion is more readily available clinically to assess the maximum toe elevation of patients, kinematic methods (such as electronic goniometers or use of a gait analysis lab when accessible) would enable identification and, more importantly, quantification of foot drop as an important obstacle to gait recovery. The subsequent treatment could then be based on novel therapies that would target the foot drop by improving CST excitability such as skilled training ${ }^{22}$ or rTMS. ${ }^{23-25}$ These therapeutic approaches could be used to increase CST excitability with the aim of decreasing foot drop and ultimately improving gait function.

\section{CONCLUSION}

These data indicate that maximum toe elevation, which reflects CST impairment, is a simple method for assessing foot drop and is an important determinant for gait function evaluated clinically.

\section{DATA ARCHIVING}

There were no data to deposit.

\section{CONFLICT OF INTEREST}

The authors declare no conflict of interest.

\section{ACKNOWLEDGEMENTS}

This research was supported by Canadian Institutes of Health Research, Danish National Research Foundation, Elsass Foundation and Bevica Foundation. Dorothy Barthélemy is a member of the Multidisciplinary sensorimotor rehabilitation research team (www.errsm.ca) supported by the CIHR and the Quebec-Ontario spinal cord injury mobility (SCI-Mob) research group funded by the Quebec Rehabilitation Research network (REPAR) and the Ontario Neurotrauma Foundation (ONF).

1 Thomas SL, Gorassini MA. Increases in corticospinal tract function by treadmil training after incomplete spinal cord injury. J Neurophysiol 2005; 94: 2844-2855.

2 Piron L, Piccione F, Tonin P, Dam M. Clinical correlation between motor evoked potentials and gait recovery in poststroke patients. Arch Phys Med Rehabil 2005; 86 1874-1878.
3 Curt A, Keck ME, Dietz V. Functional outcome following spinal cord injury: significance of motor-evoked potentials and ASIA scores. Arch Phys Med Rehabil 1998; 79 $81-86$.

4 Wirth B, van Hedel HJ, Curt A. Ankle paresis in incomplete spinal cord injury: relation to corticospinal conductivity and ambulatory capacity. J Clin Neurophysiol 2008; 25 210-217.

5 Nathan PW. Effects on movement of surgical incisions into the human spinal cord. Brain 1994; 117 (Pt 2), 337-346.

6 Calancie B, Alexeeva N, Broton JG, Suys S, Hall A, Klose KJ. Distribution and latency of muscle responses to transcranial magnetic stimulation of motor cortex after spinal cord injury in humans. J Neurotrauma 1999; 16: 49-67.

7 Westhout FD, Pare LS, Linskey ME. Central causes of foot drop: rare and underappreciated differential diagnoses. J Spinal Cord Med 2007; 30: 62-66.

8 Barthelemy D, Willerslev-Olsen M, Lundell H, Conway BA, Knudsen $H$, Biering Sorensen $\mathrm{F}$ et al. Impaired transmission in the corticospinal tract and gait disability in spinal cord injured persons. J Neurophysiol 2010; 104: 1167-1176.

9 Kirshblum SC, Burns SP, Biering-Sorensen F, Donovan W, Graves DE, Jha A et al. International standards for neurological classification of spinal cord injury (revised 2011). J Spinal Cord Med 2011; 34: 535-546.

10 Ditunno JF Jr, Barbeau H, Dobkin BH, Elashoff R, Harkema S, Marino RJ et al. Validity of the walking scale for spinal cord injury and other domains of function in a multicenter clinical trial. Neurorehabil Neural Repair 2007; 21: 539-550.

11 van Hedel HJ, Wirz M, Dietz V. Assessing walking ability in subjects with spinal cord injury: validity and reliability of 3 walking tests. Arch Phys Med Rehabil 2005; 86 190-196.

12 Ditunno JF, Scivoletto G, Patrick M, Biering-Sorensen F, Abel R, Marino R. Validation of the walking index for spinal cord injury in a US and European clinical population. Spinal Cord 2008; 46: 181-188.

13 Wirz M, Muller R, Bastiaenen C. Falls in persons with spinal cord injury: validity and reliability of the Berg Balance Scale. Neurorehabil Neural Repair 2010; 24: 70-77.

14 Lemay JF, Nadeau S. Standing balance assessment in ASIA D paraplegic and tetraplegic participants: concurrent validity of the Berg Balance Scale. Spinal Cord 2010: 48: 245-250.

15 Newstead AH, Hinman MR, Tomberlin JA. Reliability of the Berg Balance Scale and balance master limits of stability tests for individuals with brain injury. J Neurol Phys Ther 2005; 29: 18-23.

16 van Hedel HJ, Wirz M, Dietz V. Standardized assessment of walking capacity after spinal cord injury: the European network approach. Neurol Res 2008; 30: 61-73.

17 Bensoussan L, Mesure S, Viton JM, Delarque A. Kinematic and kinetic asymmetries in hemiplegic patients' gait initiation patterns. J Rehabil Med 2006; 38: 287-294.

18 Chin R, Hsiao-Wecksler ET, Loth E, Kogler G, Manwaring SD, Tyson SN et al. A pneumatic power harvesting ankle-foot orthosis to prevent foot-drop. J Neuroeng Rehabil 2009; 6: 19.

19 Weber DJ, Stein RB, Chan KM, Loeb GE, Richmond FJ, Rolf R et al. Functional electrical stimulation using microstimulators to correct foot drop: a case study. Can J Physiol Pharmacol 2004; 82: 784-792.

20 Novak AC, Olney SJ, Bagg S, Brouwer B. Gait changes following botulinum toxin A treatment in stroke. Top Stroke Rehabil 2009; 16: 367-376.

21 Paoloni M, Mangone M, Scettri P, Procaccianti R, Cometa A, Santilli V. Segmental muscle vibration improves walking in chronic stroke patients with foot drop: a randomized controlled trial. Neurorehabil Neural Repair 2009; 24: 254-262.

22 Perez MA, Lungholt BK, Nyborg K, Nielsen JB. Motor skill training induces changes in the excitability of the leg cortical area in healthy humans. Exp Brain Res 2004; 159: 197-205

23 Chen R, Classen J, Gerloff C, Celnik P, Wassermann EM, Hallett M et al. Depression of motor cortex excitability by low-frequency transcranial magnetic stimulation. Neurology 1997: 48: 1398-1403.

24 Mansur CG, Fregni F, Boggio PS, Riberto M, Gallucci-Neto J, Santos CM et al. A sham stimulation-controlled trial of rTMS of the unaffected hemisphere in stroke patients. Neurology 2005; 64: 1802-1804.

25 Belci M, Catley M, Husain M, Frankel HL, Davey NJ. Magnetic brain stimulation can improve clinical outcome in incomplete spinal cord injured patients. Spinal Cord 2004; 42: 417-419. 